\title{
Negotiation Among Autonomous Computational Agents
}

\author{
Fernando Lopes ${ }^{1}$, Nuno Mamede ${ }^{2}$, A. Q. Novais ${ }^{1}$ and Helder Coelho ${ }^{3}$ \\ ${ }^{1}$ INETI, DMS, Est. Paço do Lumiar, 1649-038 Lisboa, Portugal \\ \{fernando.lopes, augusto.novais\}@ineti.pt \\ ${ }^{2}$ IST, Avenida Rovisco Pais, 1049-001 Lisboa, Portugal \\ Nuno.Mamede@acm.org \\ ${ }^{3}$ UL, FC, DI, Campo Grande, 1700 Lisboa, Portugal \\ hcoelho@di.fc.ul.pt
}

\begin{abstract}
Autonomous agents are being increasingly used in a wide range of applications. Most applications involve or require multiple agents operating in complex environments and, over time, conflicts inevitably occur among them. Negotiation is the predominant process for resolving conflicts. Recent interest in electronic commerce has also given increased importance to negotiation. This paper presents a generic negotiation model for autonomous agents that handles multi-party, multi-issue and repeated rounds. The model is based on computationally tractable assumptions.
\end{abstract}

\section{Introduction}

Autonomous agents operate in complex environments and, over time, conflicts inevitably occur among them. Conflict resolution is crucial for achieving coordination. The predominant process for resolving conflicts is negotiation. Recent interest in electronic commerce has also given increased importance to negotiation. This paper presents a generic negotiation model for autonomous agents that handles multi-party, multi-issue, and repeated rounds. The components of the model are: (i) a prenegotiation model, (ii) a multilateral negotiation protocol, (iii) an individual model of the negotiation process, (iv) a set of negotiation strategies, and (v) a set of negotiation tactics. The model is based on computationally tractable assumptions.

This paper builds on our previous work [7, 8, 9, 10]. In these papers, we presented the prenegotiation model, introduced the individual model of the negotiation process, and defined a number of negotiation tactics. In this paper, we present a multilateral negotiation protocol, continue the description of the individual model and introduce a set of negotiation strategies.

The remainder of the paper is structured as follows. Section 2 presents a generic model of individual behavior for autonomous agents. The model forms a basis for the development of negotiating agents. Section 3 presents a generic model of negotiation for autonomous agents. Finally, related work and concluding remarks are presented in sections 4 and 5 , respectively. 


\section{Autonomous Agents}

Let Agents be a set of autonomous agents. This section briefly describes the features of every agent $a g_{i} \in$ Agents (see [7,8] for an in-depth discussion).

The agent $a g_{i}$ has a set $B_{i}=\left\{b_{i 1}, \ldots\right\}$ of beliefs and a set $G_{i}=\left\{g_{i 1}, \ldots\right\}$ of goals. Beliefs represent information about the world and the agent himself. Goals represent world states to be achieved.

The agent $a g_{i}$ has a library $P L_{i}=\left\{p t_{i 11}, \ldots\right\}$ of plan templates representing simple procedures for achieving goals. A plan template $p t_{i k l} \in P L_{i}$ is a 6-tuple that includes a header, a type, a list of conditions, a body, a list a constraints, and a list of statements [8]. The header is a 2-tuple: header ${ }_{i k l}=\left\langle\right.$ pname $_{i k l}$, pvars $\left._{i k l}\right\rangle$, where pname $_{i k l}$ is the name of $p t_{i k l}$ and pvars $_{i k l}$ is a set of variables. The library $P L_{i}$ has composite plan templates specifying the decomposition of goals into more detailed subgoals, and primitive plan templates specifying actions directly executable by $a g_{i}$.

The agent $a g_{i}$ is able to generate complex plans from the simpler plan templates stored in the library. A plan $p_{i k}$ for achieving a goal $g_{i k} \in G_{i}$ is a 3-tuple: $p_{i k}=<P T_{i k}, \leq_{h}, \leq_{t}>$, where $P T_{i k} \subseteq P L_{i}$ is a list of plan templates, $\leq_{h}$ is a binary relation establishing a hierarchy on $P T_{i k}$, and $\leq_{t}$ is another binary relation establishing a temporal order on $P T_{i k}$. The plan $p_{i k}$ is represented as a hierarchical and temporally constrained And-tree. Plan generation is an iterative procedure of: (i) plan retrieval, (ii) plan selection, (iii) plan addition, and (iv) plan interpretation [8].

At any instant, the agent $a g_{i}$ has a number of plans for execution. These plans are the plans adopted by $a g_{i}$ and are stored in the intention structure $I S_{i}=\left[p_{i 1}, \ldots\right]$. For each plan template $p t_{i k l}$ in $p_{i k}$, the header of $p t_{i k l}$ is referred as intention int $i_{i k l}$.

The agent $a g_{i}$ often has information about the agents in Agents. This information is stored in the social description $S D_{i}=\left\{S D_{i}\left(a g_{1}\right), \ldots\right\}$. Each entry $S D_{i}\left(a g_{j}\right)=\left\langle B_{i}\left(a g_{j}\right), G_{i}\left(a g_{j}\right), I_{i}\left(a g_{j}\right)\right\rangle$, contains the beliefs, goals and intentions that $a g_{i}$ believes $a g_{j}$ has.

\section{The Negotiation Model}

Let $A g=\left\{a g_{1}, \ldots, a g_{i}, \ldots, a g_{n}\right\}, A g \subseteq$ Agents, be a set of autonomous agents. Let $P_{A g}=\left\{p_{11}, \ldots, p_{i k}, \ldots, p_{n n}\right\}$ be a set of plans of the agents in $A g$ including intentions $I_{A g}=\left\{\right.$ int $_{111}, \ldots$, int $_{i k m}, \ldots$, int $\left._{n n n}\right\}$, respectively. Let the intentions in $I_{A g}$ represent commitments to achieve exclusive world states. In this situation, there is a conflict among the agents in $\mathrm{Ag}$. This section presents a domain-independent description of a computational model of negotiation. 


\subsection{Preparing and Planning for Negotiation}

The prenegotiation model defines the main tasks that each agent $a g_{i} \in A g$ must attend to in order to prepare and plan for negotiation. A brief description of these tasks follows (see [9] for an in-depth discussion).

Negotiation Problem Structure Generation. A negotiation problem $N P_{i k}$ from the perspective of $a g_{i}$ is a 6-tuple: $N P_{i k}=\left\langle a g_{i}, B_{i}, g_{i k}, i n t_{i k m}, A, I_{A}\right\rangle$, where $B_{i}$ is a set of beliefs, $g_{i k} \in G_{i}$ is a goal, $p_{i k} \in P_{A g}$ is a plan of $a g_{i}$ for achieving $g_{i k}$, int $i_{i k m} \in I_{A g}$ is an intention of $p_{i k}, A=A g-\left\{a g_{i}\right\}$ and $I_{A}=I_{A g}-\left\{i n t_{i k m}\right\}$. The problem $N P_{i k}$ has a structure NPstruct ${ }_{i k}$ consisting of a hierarchical And-Or tree. Formally, $N P$ struct $_{i k}$ is a 4-tuple: $N P$ struct $_{i k}=\left\langle N P T_{i k}, \leq_{h}, \leq_{t}, \leq_{a}\right\rangle$, where $N P T_{i k} \subseteq P L_{i}$ is a list of plan templates, $\leq_{h}$ and $\leq_{t}$ have the meaning just specified, and $\leq_{a}$ is a binary relation establishing alternatives among the plan templates in $N P T_{i k}$. The nodes of the And-Or tree are plan templates. The header of the root node describes the negotiation goal $g_{i k}$.

The structure NPstruct ${ }_{i k}$ is generated from plan $p_{i k}$ by an iterative procedure involving: (i) problem structure interpretation, (ii) plan decomposition, (iii) goal selection, (iv) plan retrieval, and (v) plan addition [9]. NPstruct ${ }_{i k}$ defines all the solutions of $N P_{i k}$ currently known by $a g_{i}$. A solution is a plan that can achieve $g_{i k}$.

Issue Identification and Prioritization. The negotiation issues of $a g_{i}$ are obtained from the leaves of $N$ struct $_{i k}$. Let $L_{i k}=\left[p t_{i k a}, \ldots\right]$ be the collection of plan templates constituting the leaves of NPstruct $t_{i k}$. The header ( pname $_{i k l}$ and pvars ${ }_{i k l}$ ) of every plan template $p t_{i k l} \in L_{i k}$ is called a fact and denoted by $f_{i k l}$. Formally, a fact $f_{i k l}$ is a 3-tuple: $f_{i k l}=\left\langle i s_{i k l}, v\left[i s_{i k l}\right], r_{i k l}\right\rangle$, where $i s_{i k l}$ is a negotiation issue (corresponding to pname $\left.i k l\right)$ ), $v\left[i s_{i k l}\right]$ is a value of $i s_{i k l}$ (corresponding to an element of pvars $i_{i k l}$ ), and $r_{i k l}$ is a list of arguments (corresponding to the remaining elements of pvars $_{i k l}$ ). Let $F_{i k}=\left\{f_{i k a}, \ldots, f_{i k z}\right\}$ be the set of facts of NPstruct $i k$. The negotiating agenda of $a g_{i}$ is the set of issues $I_{i k}=\left\{i s_{i k a}, \ldots, i s_{i k z}\right\}$ associated with the facts in $F_{i k}$. The interval of legal values for each issue $i s_{i k l} \in I_{i k}$ is represented by $D_{i k l}=\left[\min _{i k l}, \max _{i k l}\right]$.

For each issue $i s_{i k l}$, let $w_{i k l}$ be a number called importance weight that represents its importance. Let $W_{i k}=\left\{w_{i k a}, \ldots, w_{i k z}\right\}$ be the set of normalized importance weights of the issues in $I_{i k}$. The priority of the issues in $I_{i k}$ is defined as their importance.

Limits and Aspirations Formulation. Limits and aspirations are formulated for each issue. The limit for issue $i s_{i k l}$ is represented by $\lim _{i k l}$ and the initial aspiration by $a_{s p^{0}}{ }_{i k l}$, with $l_{i m}{ }_{i k l}, a p^{o}{ }_{i k l} \in D_{i k l}$ and $l_{i m} i_{i k l} \leq a s p^{0}{ }_{i k l}$.

Negotiation Constraints Definition. Constraints are defined for each issue $i s_{i k l} \in I_{i k}$. Hard constraints are linear constraints that specify threshold values for issues. They 
cannot be relaxed. The hard constraint $h c_{i k l}$ for $i s_{i k l}$ has the form: $h c_{i k l}=\left(i s_{i k l} \geq \lim _{i k l}\right.$, flex=0), where flex $=0$ represents null flexibility (inflexibility). Soft constraints are linear constraints that specify minimum acceptable values for issues. They can be relaxed. The soft constraint $s c_{i k l}$ for $i s_{i k l}$ has the form: $s c_{i k l}=\left(i s_{i k l} \geq a s p^{0}{ }_{i k l}\right.$, flex=n), where flex $=n, n \in N$, represents the degree of flexibility of $s c_{i k l}$.

Negotiation Strategy Selection. The agent $a g_{i}$ has a library $S L_{i}=\left\{s t r_{i 1}, \ldots\right\}$ of negotiation strategies and a library $T L_{i}=\left\{\right.$ tact $\left._{i 1}, \ldots\right\}$ of negotiation tactics. Negotiation strategies are functions that define the tactics to be used at the beginning and during the course of negotiation (see subsection 3.4). Negotiation tactics are functions that define the moves to be made at each point of the negotiation process (see subsection 3.5). Strategy selection is an important task and must be carefully planned [3, 12, 13]. In this paper, we assume that $a g_{i}$ selects a strategy $s t r_{i k} \in S L_{i}$ accordingly to his experience.

\subsection{A Multilateral Negotiation Protocol}

The protocol defines the set of possible tasks that each agent $a g_{i}$ can perform at each point of the negotiation process. A negotiation strategy specifies a task from the set of possible tasks. A global description of the negotiation process follows.

The process starts with $a g_{i}$ communicating a negotiation proposal prop $_{i k m}$ to all the agents in $A=A g-\left\{a g_{i}\right\}$. A negotiation proposal is a set of facts (see subsection 3.3). Each agent $a g_{j} \in A$ receives prop $_{i k m}$ and may decide either: (i) to accept prop $_{i k m}$, (ii) to reject prop $_{i k m}$ without making a critique, or (iii) to reject prop $_{i k m}$ and making a critique. A critique is a statement about issue priorities.

The process of negotiation proceeds with $a g_{i}$ receiving the responses of all the agents in $A$. Next, $a g_{i}$ checks whether a negotiation agreement was reached. If the proposal prop $_{i k m}$ was accepted by all the agents in $A$, the negotiation process ends successfully. In this case, $a g_{i}$ informs the agents in $A$ that an agreement was reached. Otherwise, $a g_{i}$ can act either: (i) by communicating a new proposal $\operatorname{prop}_{i k m+1}$, or (ii) by acknowledging the receipt of all the responses.

The process continues with the agents in $A$ receiving the response of $a g_{i}$. If $a g_{i}$ decides to communicate a new proposal $\operatorname{prop}_{i k m+1}$, each agent $a g_{j} \in A$ may again decide: (i) to accept $\operatorname{prop}_{i k m+1}$, or (ii) to reject $\operatorname{prop}_{i k m+1}$ without making a critique, or (iii) to reject prop $_{i k m+1}$ and making a critique. If $a g_{i}$ decides to acknowledge the receipt of the responses, the process continues to a new round in which another agent $a g_{k} \in A g$ communicates a proposal to all the agents in $A_{k}=A g-\left\{a g_{k}\right\}$. This is repeated for other agents in $\mathrm{Ag}$. 


\subsection{The Negotiation Process (Individual Perspective)}

The individual model of the negotiation process specifies the tasks that each agent must perform in order to negotiate in a competent way. These tasks (or processes) are shown in Fig. 1 for the specific case of an agent $a g_{i} \in A g$ that communicates a negotiation proposal. Let $N P_{i k}$ represent $a g_{i}$ 's perspective of a negotiation problem and $N$ Pstruct ${ }_{i k}$ be the structure of $N P_{i k}$. A description of the main processes follows.

Negotiation Proposal Generation. This process generates the set of initial negotiation proposals $I N P S_{i k}$ satisfying the requirements imposed by NPstruct ${ }_{i k}$. The generation of $I N P S_{i k}$ is performed through an iterative procedure involving: (i) problem interpretation, (ii) proposal preparation, and (iii) proposal addition [10]. In brief, problem interpretation consists of searching $N P s_{\text {stuct }}{ }_{i k}$ for any solution $p_{i k}$ of $N P_{i k}$ and selecting the primitive plan templates $p p t_{i k}=\left\{p t_{i k a}, \ldots, p t_{i k p}\right\}$ of $p_{i k}$. Proposal preparation consists of determining a negotiation proposal prop $p_{i k m}=\left\{f_{i k a}, \ldots, f_{i k p}\right\}$, i.e., a set of facts corresponding to the headers of the plan templates in $p p t_{i k}$. Proposal addition consists of adding prop $_{i k m}$ to $I N P S_{i k}$.

The preparation of a proposal prop $_{i k m}$ partitions the set $F_{i k}$ of facts into: (i) subset prop $_{i k m}$, and (ii) subset compl $_{i k m}=\left\{f_{i k p+1}, \ldots, f_{i k z}\right\}$, called proposal complement of prop $_{i k m}$. The facts in prop $_{i k m}$ are fundamental for achieving the negotiation goal $g_{i k}$. They are the inflexible facts of negotiation, for proposal prop $_{i k m}$. The negotiation issues $\operatorname{Iprop}_{i k m}=\left\{i s_{i k a}, \ldots, i s_{i k p}\right\}$ associated with these facts are the inflexible issues. On the other hand, the facts in compl $_{i k m}$ are not important for achieving $g_{i k}$. They are the flexible facts of negotiation, for proposal propikm. The issues Icompl $_{i k m}=\left\{i s_{i k p+1}, \ldots, i s_{i k z}\right\}$ associated with these facts are the flexible issues.

Feasible and Acceptable Proposal Preparation. This process generates the set of feasible proposals $I F P S_{i k}, I F P S_{i k} \subseteq I N P S_{i k}$, and the set of acceptable proposals IAPS $S_{i k}$, $I A P S_{i k} \subseteq I F P S_{i k}$. Let $\operatorname{prop}_{i k m}=\left\{f_{i k a}, \ldots, f_{i k p}\right\}$ be a negotiation proposal. Let Iprop $_{i k m}=\left\{i s_{i k a}, \ldots, i s_{i k p}\right\}$ be the set of issues associated with the facts in prop $_{i k m}$. Let HCprop $_{i k m}=\left\{h c_{i k a}, \ldots, h c_{i k p}\right\}$ and $S C \operatorname{prop}_{i k m}=\left\{s c_{i k a}, \ldots, s c_{i k p}\right\}$ be the sets of hard and soft constraints for issues in Iprop $_{i k m}$, respectively. A negotiation proposal prop $_{i k m} \in I N P S_{i k}$ is feasible if the issues in Iprop $_{i k m}$ satisfy the set HCprop Hkm $_{\text {of }}$ hard constraints. A feasible proposal prop $_{i k m}$ is acceptable if the issues in Iprop $_{i k m}$ satisfy the set SCprop $_{i k m}$ of soft constraints.

Feasible Proposal Evaluation. This process computes a score for each proposal in $I F P S_{i k}$ using an additive scoring function and orders the proposals in descending order of preference. Let $W_{i k}=\left\{w_{i k a}, \ldots, w_{i k p}\right\}$ be the set of importance weights of the issues in Iprop $_{i k m}$. Let $C_{i k m}=\left(v\left[i s_{i k a}\right], \ldots, v\left[i s_{i k p}\right]\right)$ be the values of the issues in Iprop $_{i k m}$ 
$\left(C_{i k m}\right.$ is called a contract). For each issue $i s_{i k l} \in \operatorname{Iprop}_{i k m}$ defined over the interval $D_{i k l}=\left[\min _{i k l}, \max _{i k l}\right]$, let $V_{i k l}$ be a component scoring function that gives the score that $a g_{i}$ assigns to a value $v\left[i s_{i k l}\right] \in D_{i k j}$ of $i s_{i k l}$. The score for contract $C_{i k m}$ is given by [13]: $V\left(C_{i k m}\right)=\sum_{j=a}^{p} \mathrm{w}_{\mathrm{ikj}} \mathrm{V}_{\mathrm{ikj}}\left(\mathrm{v}\left[\mathrm{is}_{\mathrm{ikj}}\right]\right)$. The proposal prop $_{i k m}$ is identified with contract $C_{i k m}$ and both have the same score.

Feasible Proposal Selection. This process selects a feasible proposal prop $_{i k m} \in I F P S_{i k}$. The negotiation strategy $s t r_{i k}$ of $a g_{i}$ dictates a tactic $\operatorname{tact}_{i k} \in T L_{i}$ to use. The tactic $\operatorname{tact}_{i k}$ specifies a particular proposal prop $_{i k m}$.

Feasible Proposal Modification. This process computes a new proposal prop $_{i k m+1}$ from a rejected proposal prop $_{i k m}$. The strategy $s t r_{i k}$ defines one or two tactics tact $_{i k}$, tact $_{i k+1} \in T L_{i}$. The tactics modify prop $_{i k m}$ to make it more acceptable.

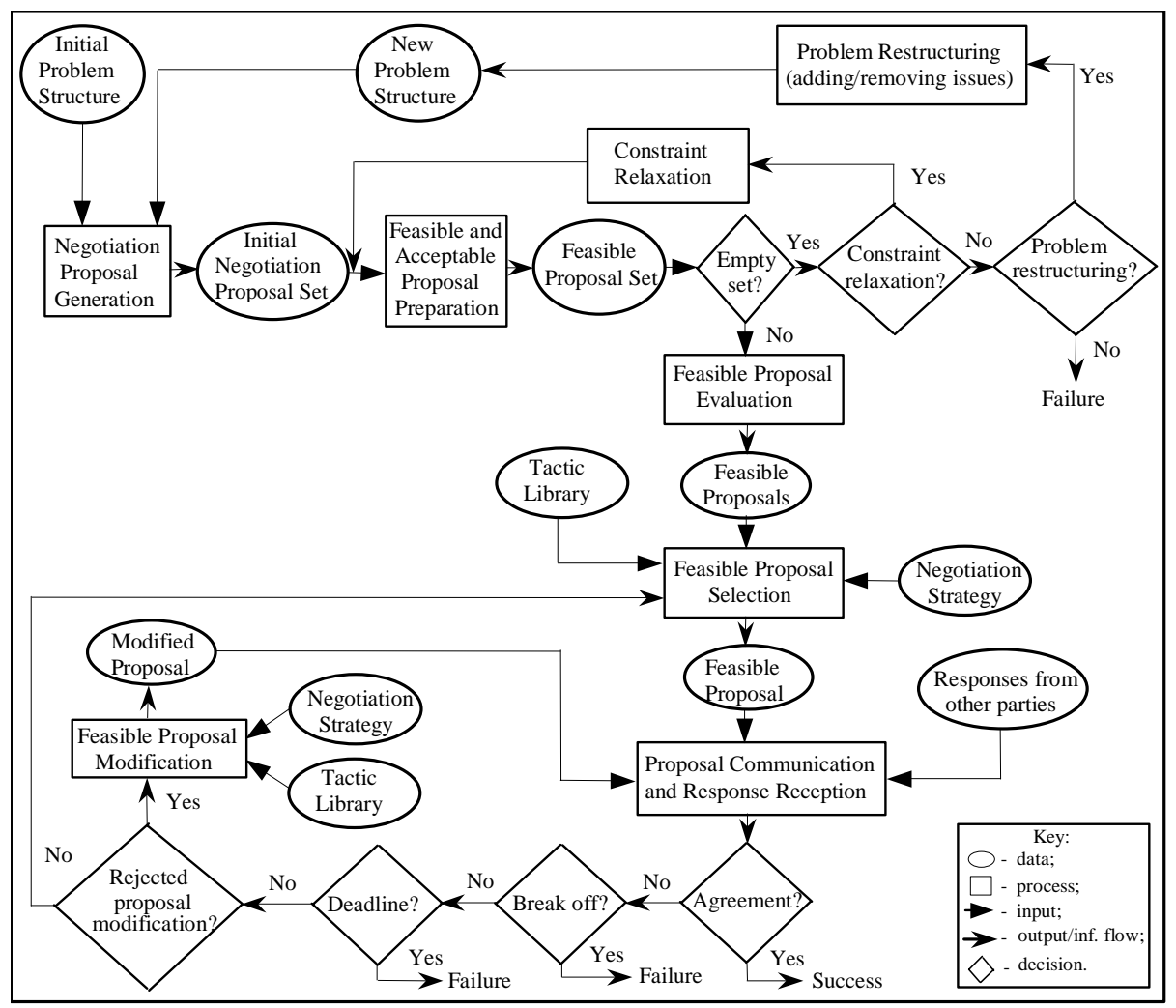

Fig. 1. The negotiation process (perspective of every agent that communicates a proposal) 


\subsection{Negotiation Strategies}

This subsection describes two classes of strategies, called concession and problem solving strategies.

Concession strategies are functions that define the opening negotiation and concession tactics. In this paper, we consider three sub-classes of strategies:

1. starting high and conceding slowly - model an optimistic opening attitude and successive small concessions;

2. starting reasonable and conceding moderately - model a realistic opening attitude and successive moderate concessions;

3. starting low and conceding rapidly - model a pessimistic opening attitude and successive large concessions.

The starting high and conceding slowly strategies are formalized by analogous functions. For instance, a strategy $\mathrm{SHOl}$ is formalized by a function:

sh_strategy_01 $\left(\right.$ state $\left., T L_{i}, F\right)=$ tact $_{i k} \mid$

$$
\begin{aligned}
& \text { if: } \text { state }=\text { "initial" then: } \text { tact }_{i k}=\text { "starting_optimistic" } \\
& \text { else: } \text { tact }_{\text {ik }}=" \text { "const_factor_tact" } \wedge F=0.1
\end{aligned}
$$

where state is the state of the negotiation, $F \in[0,1]$ is the concession factor, tact $_{i k}$ is the tactic specified by the strategy, starting_optimistic is an opening negotiation tactic, and const_factor_tact is a constant concession factor tactic (see subsection 3.5). The strategies in the other subclasses are formalized by similar functions.

Problem solving strategies define the opening negotiation, concession and compensation tactics. In this paper, we consider two sub-classes of strategies:

1. low priority concession making - model a realistic opening attitude, large concessions on issues of low priority and small concessions on other issues;

2. low priority concession making with compensation - these strategies are similar to previous strategies; however, concessions are interleaved with compensations.

Low priority concession making strategies partition the set $I_{i k}$ of issues into: (i) subset $I_{i k+}$, corresponding to higher priority issues, and (ii) subset $I_{i k \text { - }}$, corresponding to the remaining issues. Again, the strategies in this sub-class are formalized by analogous functions. For instance, a strategy $\mathrm{LPO} 1$ is formalized by a function:

$$
\begin{aligned}
& \text { lp_strategy_01 }\left(\text { state } T L_{i}, I_{i k}, F_{1}, F_{2}\right)=\left(\text { tact }_{i k}, I_{i k+}, \text { tact }_{i k+1}, I_{i k-}\right) \mid \\
& \text { if: state ="initial" then: } \text { tact }_{i k}=\text { "starting_realistic" } \wedge \text { tact }_{i k+1}=\text { "nil" } \\
& \text { else: } I_{i k}=I_{i k+}+I_{i k^{-}} \wedge \forall i t_{i k j} \in I_{i k+}, \text { tact }_{i k}=\text { "const_factor_tact" } \wedge F_{l}=0.10 \wedge \\
& \forall i t_{i k j} \in I_{i k,}, \text { tact }_{i k+1}=" \text { const_factor_tact" } \wedge F_{2}=0.35
\end{aligned}
$$

where state and const_factor_tact have the meaning just specified, $F_{1}$ and $F_{2}$ are constants, tact $_{i k}$ and tact $_{i k+1}$ are the tactics defined by the strategy, and starting_realistic is an opening negotiation tactic (see subsection 3.5). The formalization of the strategies in the other sub-class is essentially identical to that. 


\subsection{Negotiation Tactics}

This section describes two classes of tactics, called opening negotiation and concession tactics.

Opening negotiation tactics specify a proposal to submit at the beginning of negotiation. Let IFPS $S_{i k}$ and IAPS $S_{i k}$ be the sets of feasible and acceptable proposals of $a g_{i}$, respectively. Let $I N A P S_{i k}=I F P S_{i k}-I A P S_{i k}$. Let $\operatorname{Vprop}_{i k h}$ be the score of proposal $\operatorname{prop}_{i k h} \in I A P S_{i k}$. Let $A_{p r o p}{ }_{i k h}$ be the set of initial aspirations of $a g_{i}$ for issues in prop $_{i k h}$ and VAprop $_{i k h}$ be the score of $A_{\text {prop }}{ }_{i k h}$. Let Dif $_{i k h}=\mid$ Vprop $_{i k h}-$ VAprop $_{i k h} \mid$. Similarly, let $\operatorname{Vprop}_{i k h+1}$ be the score of proposal $\operatorname{prop}_{i k h+1} \in I N A P S_{i k}$. Let Aprop $_{i k h+1}$ be the set of initial aspirations of $a g_{i}$ for issues in prop $i k h+1$ and VAprop $_{i k h+1}$ be the score of Apropo $_{i k h+1}$. Let Dif $f_{i k h+1}=\mid$ Vprop $_{i k h+1}-$ VAprop $_{i k h+1} \mid$. We consider three tactics:

1. starting optimistic - specifies the proposal prop $_{i k 1}$ with the highest score;

2. starting realistic - specifies either: (i) proposal prop $_{i k h}$ with the lowest score, if $D i f_{i k h} \leq D i f_{i k h+1}$, or (ii) proposal $\operatorname{prop}_{i k h+1}$ with the highest score, if $D i f_{i k h}>D i f_{i k h+1}$;

3. starting pessimistic - specifies the proposal rrop $_{i k n}$ with the lowest score.

The three tactics are formalized by similar functions. For instance, the tactic starting optimistic is formalized by the following function:

$$
\text { starting_optimistic }\left(I F P S_{i k}\right)=\operatorname{prop}_{i k 1} \mid \forall \operatorname{prop}_{i k j} \in I F P S_{i k}, \operatorname{Vprop}_{i k 1} \geq \operatorname{Vprop}_{i k j}
$$

Concession tactics are functions that compute new values for each issue. In this paper, we consider two sub-classes of tactics: (i) constant concession factor tactics, and (ii) total concession dependent tactics. In each sub-class, we consider five tactics:

1. stalemate - models a null concession on $i s_{i k j}$;

2. tough - models a small concession on $i s_{i k j}$;

3. moderate - models a moderate concession on $i s_{i k j}$;

4. soft - models a large concession on $i s_{i k j}$;

5. compromise - models a complete concession on $i s_{i k j}$.

Let prop $_{i k m}$ be a proposal submitted by $a g_{i}$ and rejected. Let $v\left[i s_{i k j}\right]_{m}$ be the value of $i s_{i k j}$ offered in $\operatorname{prop}_{i k m}$. Let $l i m_{i k j}$ be the limit for $i s_{i k j}$. Let $v\left[i i_{i k j}\right]_{m+1}$ be the new value of $i s_{i k j}$ to be offered in a new proposal prop $i k m+1$. Let $V_{i k j}$ be the component scoring function for $i s_{i k j}$. The constant concession factor tactics are formalized by a function const_factor_tact which takes $v\left[i s_{i k j}\right]_{m}$, a constant $w, \lim _{i k j}$ and another constant cte as input and returns $v\left[i s_{i k j}\right]_{m+1}$, i.e., 


$$
\begin{aligned}
& \text { const_factor_tact }\left(v\left[i s_{i k j}\right]_{m}, w, l i m_{i k j}, \text { cte }\right)=v\left[i s_{i k j}\right]_{m+1} \mid \\
& \qquad v\left[i s_{i k j}\right]_{m+1}=v\left[i s_{i k j}\right]_{m}+(-1)^{w} F\left|l i m_{i k j}-v\left[i s_{i k j}\right]_{m}\right| \wedge F=c t e
\end{aligned}
$$

where $w=0$ if $V_{i k j}$ is monotonically decreasing or $w=1$ if $V_{i k j}$ is monotonically increasing and $F$ is the concession factor. The five tactics are defined as follows: the stalemate tactic by $F=0$, the tough tactic by $F \in] 0,0.2]$, the moderate tactic by $F \in] 0.2,0.3]$, the soft tactic by $F \in] 0.3,0.4]$, and the compromise tactic by $F=1$.

The total concession dependent tactics are similar to the previous tactics, but $F$ is a function of the total concession. Let $v\left[i s_{i k j}\right]_{0}, \ldots, v\left[i s_{i k j}\right]_{m}$, be the values of $i s_{i k j}$ successively offered by $a g_{i}$, with $V_{i k j}\left(v\left[i s_{i k j}\right]_{h-1}\right) \geq V_{i k j}\left(v\left[i s_{i k j}\right]_{h}\right), 0 \leq h \leq m$. The total concession Ctotal made by $a g_{i}$ on $i s_{i k j}$ is: Ctotal $=\left|v\left[i s_{i k j}\right]_{O^{-}} v\left[i s_{i k j}\right]_{m}\right|$. These tactics are formalized by a function $t c d \_t a c t i c s$ which takes $v\left[i s_{i k j}\right]_{m}, w, \lim _{i k j}$, a constant $\lambda \in R^{+}$, Ctotal and $v\left[i s_{i k j}\right]_{0}$ as input and returns $v\left[i s_{i k j}\right]_{m+1}$, i.e.,

$$
\begin{array}{r}
\operatorname{tcd} \_ \text {tactics }\left(v\left[i s_{i k j}\right]_{m}, w, \lim _{i k j}, \lambda, C t o t a l, v\left[i s_{i k j}\right]_{O}\right)=v\left[i s_{i k j}\right]_{m+1} \mid \\
v\left[i s_{i k j}\right]_{m+1}=v\left[i s_{i k j}\right]_{m}+(-1)^{w} F\left|\lim _{i k j}-v\left[i s_{i k j}\right]_{m}\right| \\
F=1-\lambda \text { Ctotal / }\left|\lim _{i k j}-v\left[i s_{i k j}\right]_{O}\right|
\end{array}
$$

\section{Related Work}

The design of negotiating agents has been investigated from both a theoretical and a practical perspective. Researchers following the theoretical perspective attempt mainly to develop formal models. Some researchers define the modalities of the mental state of the agents, develop a logical model of individual behavior, and then use the model as a basis for the development of a formal model of negotiation or argumentation (e.g., [6]). However, most researchers are neutral with respect to the modalities of the mental state and just develop formal models of negotiation (e.g., [5]). Generally speaking, most theoretical models are rich but restrictive. They made assumptions that severely limit their applicability to solve real problems.

Researchers following the practical perspective attempt mainly to develop computational models, i.e., models specifying the key data structures and the processes operating on these structures. Some researchers start with a model of individual behavior, develop or adopt a negotiation model, and then integrate both models (e.g., [11]). Again, most researchers prefer to be neutral about the model of individual behavior and just develop negotiation models (e.g., [1]). Broadly speaking, most computational models are based on ad hoc principles. They lack a rigorous theoretical grounding. Despite these, some researchers believe that it is necessary to develop computational models in order to use agents in real-world applications [14]. Accordingly, we developed a computational negotiation model.

As noted, most researchers have paid little attention to the problem of how to integrate models of individual behavior with negotiation models. However, it is one of the costliest lessons of computer science that independently developed components resist subsequent integration in a smoothly functioning whole [2]. Accordingly, we developed a model that accounts for a tight integration of the individual capability of planning and the social capability of negotiation. 
We are interested in negotiation among both self-motivated and cooperative agents. Our structure for representing negotiation problems is similar to decision trees and goal representation trees [4], but there are important differences. Our approach does not require the quantitative measures typical of decision analysis. Also, our approach is based on plan templates and plan expansion, and not on production rules and forward or backward chaining. In addition, our formulae for modeling concession tactics are similar to the formulae used by Faratin et al. [1]. Again, there are important differences. The total concession criterion is not used by other researchers and our formulae: (i) assure that the agents do not negotiate in bad faith, and (ii) model important experimental conclusions about human negotiation.

\section{Discussion and Future Work}

This article has introduced a computational negotiation model for autonomous agents. There are several features of our work that should be highlighted. First, the model is generic and can be used in a wide range of domains. Second, the structure of a negotiation problem allows the direct integration of planning and negotiation. Also, this structure defines the set of negotiation issues. Third, the model supports problem restructuring ensuring a high degree of flexibility. Problem restructuring allows the dynamic addition of negotiation issues. Finally, the negotiation strategies are motivated by human negotiation procedures [3, 12]. Our aim for the future is: (i) to extend the model, and (ii) to finish the experimental validation of the model.

\section{References}

1. Faratin, P., C. Sierra, N. Jennings, "Negotiation Decision Functions for Autonomous Agents", Robotics and Autonomous Systems, 24(3-4), 1998, 159-182.

2. Hayes-Roth, B. "On Building Integrated Cognitive Agents: A Review of Allen Newell's Unified Theories of Cognition." Artif. Intell., 59(1-2), 1992, 329-341.

3. Lewicki, R., D. Saunders, J. Minton, Neg. Readings, Exercises-Cases, McGraw Hill, 1999.

4. Kersten G., W. Michalowski, S. Szpakowicz Z. Koperczak, 1991, "Restruturable Representations of Negotiation”, Management Science, 37(10), 1991, 1269-1290.

5. Kraus, S., J. Wilkenfeld, G. Zlotkin, "Multiagent Negotiation Under Time Constraints", Artif. Intell., 75, 1995, 297-345.

6. Kraus, S., K. Sycara, A. Evenchik, "Reaching Agreements Through Argumentation: a Logical Model and Implementation", Artif. Intell., 104, 1998, 1-69.

7. Lopes, F., N. Mamede, H. Coelho, A. Q. Novais, "A Negotiation Model for Intentional Agents", In Multi-Agent Systems in Production, Elsevier Science, 1999, 211-216.

8. Lopes, F., N. Mamede, A. Q. Novais, H. Coelho, "Towards a Generic Negotiation Model for Intentional Agents", In Agent-Based Inf. Systems, IEEE Comp. Press, 2000, 433-439.

9. Lopes, F., N. Mamede; A. Q. Novais, H. Coelho, "Conflict Management and Negotiation Among Intentional Agents", In Agent-Based Simulation, SCS-Europe, 2001, 117-124.

10. Lopes, F., N. Mamede, A. Q. Novais, H. Coelho, "Negotiation Tactics for Autonomous Agents." In Internet Robots, Systems, Applications. IEEE Comp. Press, 2001, 708-714.

11. Muller, J., The Design of Intelligent Agents, Springer-Verlag, 1996 (LNAI 1177).

12. Pruitt, D., Negotiation Behavior, Academic Press, 1981.

13. Raiffa, H., The Art and Science of Negotiation, Harvard University Press, 1982.

14. Rao, A. "Integrated Agent Architecture: Execution and Recognition of Mental-States." In Distrib. Artif. Intell. Archit. Modelling, Springer Verlag, 1995, 159-173 (LNAI 1087). 